\title{
Uso da aproximação fractal no ajuste da curva de retenção de água no solo
}

\author{
Francisco J. R. da Paixão ${ }^{1}$, Antonio R. S. de Andrade ${ }^{2}$, Carlos A. V. de Azevedo ${ }^{3}$, Vera L. A. de Lima ${ }^{3} \&$ José Dantas Neto ${ }^{3}$
}

\begin{abstract}
RESUMO
Objetivou-se, neste trabalho, explorar a aplicabilidade da teoria de fractais para estimar a curva de retenção de água no solo através do modelo proposto por Brooks \& Corey (1964) modificado por Pierrer et al. (1996), utilizando-se a dimensão fractal com base em dados medidos da distribuição das partículas e do tamanho dos poros do solo. O estudo foi realizado em um solo cultivado com gergelim e irrigado com sistema de irrigação por aspersão convencional. As dimensões $D_{S W R C}$ e $D_{\text {PSD }}$, que são medidas fractais representativas da distribuição do tamanho dos poros e das partículas do solo, respectivamente, foram definidas como ferramentas descritivas para estimativa da curva de retenção de água no solo. Amostras do solo foram coletadas para as profundidades de 0-20, 20-40 e 40-60 cm, totalizando 36 pontos amostrais. Houve variação entre os modelos definidos pelas dimensões fractais $D_{S W R C}$ e $D_{\text {PSD }}$, e nas diferentes profundidades avaliadas. Verificou-se que o modelo de Brooks \& Corey (1964), modificado com uso da aproximação fractal baseada nos teores de água no solo em função do tamanho dos poros $\left(\mathrm{BCD}_{\text {SWRC }}\right.$ ), é simples em sua utilização e capaz de predizer satisfatoriamente tanto a curva de retenção como a água disponível no solo.
\end{abstract}

Palavras-chave: física do solo, tamanho dos poros, manejo de água

\section{Use of fractal approach for the adjustment of the soil water retention curve}

\begin{abstract}
The objective in this work was to explore the applicability of fractal theory to estimate the soil water retention curve, through the model proposed by Brooks \& Corey (1964), modified by Pierrer (1996), using the fractal dimension based on observed data of the distribution of particles and pore sizes. The study was accomplished in a soil cultivated with sesame and irrigated with a conventional sprinkle irrigation system. The dimensions $D_{\mathrm{WRC}}$ and $\mathrm{D}_{\text {PSD }}$, which are fractal measures representative of the distribution of the size of the pores and of the particles of the soil, respectively, were defined as descriptive tools for estimating the soil water retention curve. Samples of the soil were collected for the depths of 0-20, 20-40 and 40-60 cm, totaling 36 samples. It was verified that the Brooks \& Corey model, modified with the use of the fractal approach based on the soil water contents as a function of the pores of the soil $\left(B C D_{S W R C}\right)$, is simple in its use and capable of predicting satisfactorily the retention curve as well as the available water in the soil.
\end{abstract}

Key words: soil physics, pores size, water management

\footnotetext{
${ }^{1}$ Doutorando em Engenharia Agrícola, UFCG. Fone: (88) 9931-8868. E-mail: jardel.paixao@gmail.com

2 UAG/UFRPE, Garanhuns, PE. E-mail: arsauag@uag.ufrpe.br

3 UAEA/UFCG, Av. Aprígio Veloso, 882, Bodocongó, CEP 58109-115, Campina Grande, PB. Fone: (83) 3310-1056. E-mail: cazevedo@deag.ufcg.edu.br; antunes@deag.ufcg.edu.br; zedantas@deag.ufcg.edu.br
} 


\section{INTRODUÇÃO}

O solo constitui um reservatório ao qual se repõe, periodicamente, a água retirada pela cultura, respeitando-se um valor limite inferior para a disponibilidade de água, porém o conhecimento deste atributo físico-hídrico do solo encerra uma complexidade de fatores inerentes a cada local de amostragem, o que leva às dificuldades da sua avaliação. Entre os principais fatores que contribuem para tal complexidade, estão os métodos utilizados na determinação deste atributo do solo, além da própria variabilidade espacial do solo. Desde o início do século XX diversas metodologias existentes para a determinação dos atributos físico-hídricos do solo têm preocupado pesquisadores em virtude de proporcionarem diferentes valores alterando, desta forma, resultados de pesquisas, mesmo com o solo considerado homogêneo em parcelas experimentais.

Segundo Coelho Filho et al. (2001) não apenas na experimentação mas também na elaboração e manejo de projetos de irrigação, é fundamental o conhecimento dos atributos físico-hídricos do solo, principalmente da curva de retenção e da água nele disponível. Muitas vezes, seja pelas dificuldades ao acesso aos laboratórios, custos altos e até negligência, não se utilizam de métodos adequados para uma caracterização mais detalhada da área irrigada, resultando em insucesso associado a uma escolha inadequada do sistema de irrigação, turno de rega e lâmina de água, dentre outros.

Conforme Carvalho et al. (2004), uma das grandes preocupações dos cientistas que trabalham com física dos solos é encontrar uma única propriedade capaz de caracterizar a maior parte das informações importantes referentes a determinada mancha de solo que se está estudando; usualmente, as pesquisas nesta área são relacionadas para distribuição do tamanho dos poros e dos agregados.

Dificuldades como essas favorecem procedimentos e modelos matemáticos sem levar em consideração a física dos atributos do solo, razão pela qual a geometria fractal se apresenta como nova ferramenta adicional, que leva em conta a dimensão fractal resultante da distribuição das partículas e do tamanho dos poros; em assim sendo, a geometria fractal se torna capaz de relacionar a dimensão fractal com o potencial matricial e a umidade do solo (Fuentes et al., 2005).

A geometria fractal foi introduzida nos anos 70, por Benoit Mandelbrot, matemático polonês que propôs, através dela, uma maneira que possibilitasse melhor capacidade de representar estruturas complexas e irregulares da natureza; ao contrário da geometria euclidiana, a fractal se apresenta como ferramenta eficientemente capaz de quantificar e qualificar série de dados temporais e espaciais descrevendo, com maior exatidão, a sua complexidade (Hott et al., 2005).

A aplicação da análise fractal tem, como principal função, focalizar a complexidade das informações espaciais, fornecendo ao especialista da área uma rigorosa abordagem para fundamentar a mensuração, a análise e a representação quantitativa e geométrica do espaço real. A representação do espaço e distribuição porosa do solo são comparações explícitas entre a complexidade da irregularidade do solo e a propriedade ideal dos elementos espaciais, por meio de uma generalização capaz de associar estes elementos à sua forma e comportamento, em uma linguagem geométrica. Em algumas ciências de cunho espacial, como ciências do solo, a análise fractal tem sido direcionada principalmente à representação das formas, tamanhos da distribuição espacial das partículas e dos espaços porosos do solo (Fuentes et al., 2001).

Em ciências do solo os fractais são utilizados para descrição do percurso de infiltração e redistribuição da água, além de modelar, de forma eficiente, a ocorrência de fenômenos durante esses dois processos, uma vez que o solo é um corpo tridimensional. A dimensão fractal pode ser determinada com alguns atributos físicos do solo, permitindo estudos com novas abordagens fundamentadas em parâmetros físicos, passando a ocupar o espaço de estudos utilizando-se parâmetros puramente empíricos (Hott et al., 2005).

Recentemente, Pierrier et al. (1996) ajustaram os dados da curva de retenção a partir de funções não-lineares estabelecidas com base na geometria fractal e com uso de relações físicas entre a dimensão fractal e o comportamento funcional das propriedades do solo, através do modelo proposto por Brooks \& Corey (1964), que mais tarde o modificaram, mediante procedimento que envolve dois passos: i) o estabelecimento de relações não-lineares entre a dimensão fractal (D) e as propriedades do solo: umidade de água na saturação $(\theta s)$, umidade residual $(\theta r)$, potencial matricial de água no solo $\left(\psi_{\mathrm{m}}\right)$ e o valor absoluto do potencial de entrada de ar no solo $\left(\psi_{\mathrm{o}}\right)$; ii) estimativa simultânea dos valores de $\theta$ s, $\theta$ r e $\psi_{\mathrm{m}}$ pela aplicação da técnica dos mínimos quadrados para ajuste de funções não-lineares e, logo após, a substituição desses valores no modelo de Brooks \& Corey (1964), modificado com uso da aproximação fractal, que estima a curva de retenção de água no solo. Esta abordagem é chamada de ENR (Extended Non - Linnear Regression).

Ante o exposto se objetiva avaliar a adequabilidade da teoria de fractais para estimar a curva de retenção de água no solo através do modelo proposto por Brooks \& Corey (1964) modificado por Pierrier et al. (1996), utilizando-se a dimensão fractal.

\section{MATERIAL E MÉTODOS}

O estudo foi realizado na estação experimental da Empresa Estadual de Pesquisas Agropecuárias da Paraíba, Lagoa Seca, PB, em área cultivada com gergelim e irrigada com sistema de irrigação por aspersão convencional. Coletaramse amostras de solo deformadas da área experimental, nas profundidades de 0-20, 20-40 e de 40-60 cm, totalizando 36 pontos amostrais; na análise granulométrica e da textura do solo, utilizaram-se os métodos do Densímetro e da Pipeta de Bouyoucos, respectivamente (Gee \& Bauder, 1986); na análise textural a dispersão foi obtida mecânica (agitação) e quimicamente, utilizando-se como dispersante o hidróxido de sódio $1 \mathrm{~N}$.

Na pesquisa, realizada no Laboratório de Física de Solo da Universidade Federal de Campina Grande, se determinaram, de início, as percentagens de areia, silte e argila do 
solo; segundo a classificação da EMBRAPA (1999), tratase de um Neosolo Reolitico; inicialmente, determinou-se a curva de distribuição de partículas do solo e, depois, sua curva de retenção.

Para avaliar o desempenho do modelo proposto por Brooks \& Corey (1964), modificado por Pierrier et al. (1996), na estimava da curva de retenção de água no solo em relação aos valores determinados em laboratório, três critérios estatísticos diferentes foram utilizados: (a) medidas estatísticas descritivas; (b) erro padrão de estimativa da média para os modelos de ajuste da curva de retenção de água no solo, nas três profundidades estudadas e (c) teste de aderência Kolmogorov-Smirnov (KS) a nível de significância de 5\% de probabilidade (Bussab \& Marettin, 2004):

\section{Metodologia para estimar a dimensão fractal}

As dimensões fractais foram estimadas por dois métodos baseados nos atributos físico-hídricos do solo: (i) distribuição dos tamanhos das partículas do solo, denominado PSD $\left(D_{\mathrm{PSD}}\right)$ e (ii) curva de retenção de água no solo, chamado SWRC ( $\left.\mathrm{D}_{\text {SWRC }}\right)$ :

\section{Dimensão fractal pelo método PSD}

Uma das metodologias capazes de determinar a dimensão fractal do solo se baseia na distribuição do tamanho médio das partículas sólidas do solo, uma vez que este tamanho e a dimensão fractal estão associados à estrutura do solo. A dimensão fractal $\mathrm{D}_{\mathrm{PSD}}$ foi estimada pelo procedimento proposto por Tyler \& Wheatcraft (1992), com base nos valores da distribuição do tamanho das partículas do solo, conforme expressão:

$W(R)=\left[\frac{\pi(1-D)}{\beta(3-D)}\left(\frac{2 \pi \rho_{S}}{\rho_{G}\left(\rho_{S} S\right)}\right)^{\frac{1-D}{2}} \frac{2\left(\rho_{S} S\right)^{1-D}}{5-3 D}\right]^{\frac{2}{D^{2}-5 D+4}} R^{\frac{3 D^{2}-13 D+14}{D^{2}-5 D+4}}$

sendo $\mathrm{W}(\mathrm{R})$ a massa acumulativa das partículas do solo, $\mathrm{S}$ é uma constante que caracteriza a forma das partículas, $\rho_{\mathrm{G}}$ densidade do solo, $\rho_{\mathrm{S}}$ é a densidade das partículas e $\mathrm{R}$ o raio médio das partículas do solo, $\mathrm{D}_{\mathrm{PSD}}$ a dimensão fractal, representativo da distribuição do tamanho das partículas do solo.

A Eq. 1 é a expressão através da qual se estima o percentual da massa acumulativa das partículas em função dos raios das partículas do solo.

No sentido de se estimar $\mathrm{D}_{\mathrm{PDS}}$, aplica-se logaritmo à Eq. 1; verifica-se que a dimensão fractal ( $\mathrm{D}_{\mathrm{PDS}}$ ) pode ser avaliada através do coeficiente angular da reta que relaciona log W(R) com log R:

$$
\log [\mathrm{W}(\mathrm{R})]=\log \mathrm{c}+\mathrm{D} \log \mathrm{R}
$$

Um valor de $\mathrm{D}=0$ representa uma distribuição de partículas composta de uma única classe; valores de D maiores que 3 refletem uma distribuição composta de partículas de vários tamanhos, dominada numericamente por pequenas partículas (Bacchi et al., 1996, Antonino et al., 2004). A dimensão fractal obtida por este procedimento, resulta na dimensão fractal de superfície baseada na distribuição do tamanho das partículas do solo (Perfect et al., 1992).

\section{Dimensão fractal pelo método SWRC}

Com base na equação proposta por Brooks \& Corey (1964), modificada por Pierrier et al. (1996), aplica-se o processo de regressão não linear aos dados de tensão de água no solo. Uma maneira mais específica da relação entre o volume de poros e o seu raio, foi encontrada por Pierrier et al. (1996):

$$
-\frac{\mathrm{dV}(\geq \mathrm{r})}{\mathrm{dr}}=\beta(\mathrm{E}-\mathrm{D}) \mathrm{r}^{\mathrm{E}-\mathrm{D}-1}
$$

em que: E representa a dimensão euclidiana, $\beta$ é uma constante e r é o raio do poro.

Para estimar o teor de água no solo assume-se que o valor do raio do poro é inversamente proporcional ao potencial hidráulico h, ou seja, $h=A / r$, em que A é uma constante; tradicionalmente, usam-se valores absolutos das tensões de água no solo, em lugar de tensões negativas; a Eq. 3 é muito usada para se estimar a curva de retenção de água no solo, mesmo havendo outras formas de se correlacionar os poros do solo com as tensões da água (Tyler \& Wheatcraft, 1990; Bird et al., 1996).

O modelo para se estimar a curva de retenção de água proveniente da Eq. 3, apresenta uma rígida relação com uma lei física, o conteúdo de água do solo e a tensão com que esta água se encontra retida entre as partículas sólidas do solo (Pierrier et al., 1996). Com base na equação proposta por Brooks \& Corey (1964), modificada por Pierrier et al. (1996), a dimensão fractal $\left(\mathrm{D}_{\text {SWRC }}\right)$ foi determinada aplicando-se o processo de regressão não linear aos dados de umidade do solo $(\theta)$ versus potencial matricial de água no solo $\left(\psi_{\mathrm{m}}\right)$ por meio da seguinte expressão:

$$
\theta(h)=\theta r+\left(\theta_{\mathrm{s}}-\theta_{\mathrm{r}}\right)\left(\frac{\mathrm{h}_{\mathrm{s}}}{\mathrm{h}}\right)^{3-\mathrm{D}_{\mathrm{swec}}}
$$

sendo $\theta_{(\mathrm{h})}$ a umidade do solo, em base de volume, na tensão h, $\theta_{\mathrm{s}}$ a umidade do solo em base de volume na saturação, $\theta_{\mathrm{r}}$ a umidade residual do solo (correspondente ao ponto de murcha permanente), $h_{\mathrm{s}}$ o valor absoluto da tensão de água no ponto de entrada de ar e $\mathrm{D}_{\text {SWRC }}$ representa a dimensão fractal da distribuição dos tamanhos de poros do solo.

Para ajuste da curva de retenção de água no solo por meio da dimensão fractal, foi editado um programa em linguagem java e, para ajuste da curva de retenção por meio do modelo de Brooks \& Corey (1964) empiricamente, usou-se o programa computacional Soil Water Retention Curve (Versão Beta 3.0).

\section{Curva de retenção de água no solo}

Para as 36 amostras do solo coletadas com estrutura indeformada e para diferentes profundidades, determinações da curva de retenção de água no solo foram realizadas por meio de dois métodos:

\section{Método de laboratório}

A curva de retenção de água no solo foi determinada em laboratório usando-se o Extrator de Umidade de Reichardt, nas tensões de 10, 33, 100, 300, 500, 1000 e $1500 \mathrm{kPa}$, segundo a metodologia descrita por Guerra (2002) e Libardi (2000). 


\section{Método indireto}

A curva de retenção de água no solo relaciona determinada tensão (potencial matricial) com a umidade do solo, refletindo a distribuição do tamanho dos poros do solo e sua sinuosidade, razão por que, em sua determinação, é imprescindível utilizar metodologias que reproduzam a verdadeira condição estrutural do solo; desta forma se estimaram os teores de água no solo em função do potencial matricial, por meio do modelo original de Brooks \& Corey (1964) e também por este modelo modificado (Eq. 4) com o uso da aproximação fractal (Pierrier et al., 1996).

\section{RESULTADOS E DISCUSSÃO}

Os valores médios das percentagens dos separados texturais para as três profundidades do solo da área em estudo, apontam textura arenosa nas profundidades de 0-20 e 20-40 cm e argilosa na profundidade de 40-60 cm, com os seguintes valores texturais médios: de $0-20 \mathrm{~cm}$ : areia $75,27 \mathrm{~g} \mathrm{~kg}^{-1}$, silte $8,08 \mathrm{~g} \mathrm{~kg}^{-1}$ e argila $16,65 \mathrm{~g} \mathrm{~kg}^{-1}$; de 20-40 cm: areia $72,24 \mathrm{~g} \mathrm{~kg}^{-1}$, silte $10,10 \mathrm{~g} \mathrm{~kg}^{-1}$ e argila 17,66 g kg-1 de 40-60 cm: areia 74,48 g kg-1$^{-1}$, silte 9,84 g kg$^{-1}$ e argila 15,68 $\mathrm{g} \mathrm{kg}^{-1}$, caracterizado como um solo franco-arenoso nessas três profundidades.
Os valores da dimensão fractal obtidos pelos dois métodos usados nesta pesquisa ( $\mathrm{D}_{\mathrm{PSD}}$ e $\mathrm{D}_{\mathrm{SWRC}}$ ), foram incorporados aos valores oriundos da curva de retenção de água do solo, através do modelo proposto por Books \& Corey (1964) modificado por Pierrier et al. (1996), capaz de ajustar a curva de retenção estimando-se, assim, os teores de água no solo em função do potencial matricial. Os resultados do resumo estatístico para os valores médios são apresentados na Tabela 1.

Conforme a Tabela 1, os baixos valores do desvio padrão (DP) e do coeficiente de variação (CV) indicam baixa variabilidade dos valores médios dos teores de água no solo, em função do potencial matricial. Os maiores valores de CV (\%) e DP foram constatados na profundidade de 40-60 cm. Esta variabilidade se deve, provavelmente, às características físicas e hídricas do solo, tais como: heterogeneidade da textura e tamanho e forma das partículas, proporcionando grande variação na umidade do solo. Verifica-se, para as três profundidades estudadas, que o $\mathrm{CV}$ indicou um acréscimo acentuado para os conteúdos de umidade correspondentes aos maiores potenciais matriciais de água no solo (superiores a $300 \mathrm{kPa}$ ), que pode ser decorrente da influência de fatores e processos de formação dos solos que têm determinado, por sua vez, variações dos separados texturais no sentido vertical. De acordo com Mayr \& Jarvis (2000), os solos arenosos são mais sensíveis

Tabela 1. Resumo estatístico dos valores médios da umidade volumétrica $\left(\mathrm{cm}^{3} \mathrm{~cm}^{-3}\right)$ do solo nas respectivas profundidades estudadas em função do potencial matricial aplicado

\begin{tabular}{|c|c|c|c|c|c|c|c|c|c|c|c|c|}
\hline \multirow{2}{*}{$\begin{array}{c}\Psi_{\mathrm{m}} \\
(\mathrm{kPa})\end{array}$} & $x$ & DP & CV & $x$ & DP & $\mathrm{CV}^{-}$ & $\bar{X}$ & DP & CV & $X$ & $\mathrm{DP}$ & CV \\
\hline & \multicolumn{3}{|c|}{ Observados } & \multicolumn{3}{|c|}{ BCD $_{\text {SWR }}$} & \multicolumn{3}{|c|}{$B C D_{P S D}$} & \multicolumn{3}{|c|}{ Brooks \& Corey (1964) } \\
\hline \multicolumn{13}{|c|}{$0-20 \mathrm{~cm}$ de profundidade } \\
\hline 10 & 0,17 & 0,02 & 14,50 & 0,17 & 0,02 & 14,50 & 0,23 & 0,0012 & 0,0354 & 0,18 & 0,02 & 14,27 \\
\hline 30 & 0,16 & 0,02 & 13,80 & 0,15 & 0,02 & 15,85 & 0,19 & 0,0002 & 0,0161 & 0,15 & 0,02 & 14,83 \\
\hline 100 & 0,14 & 0,02 & 16,10 & 0,13 & 0,02 & 17,33 & 0,16 & 0,0004 & 0,0210 & 0,13 & 0,02 & 15,06 \\
\hline 500 & 0,10 & 0,01 & 16,44 & 0,10 & 0,02 & 19,34 & 0,12 & 0,0001 & 0,0122 & 0,11 & 0,01 & 14,90 \\
\hline 1000 & 0,09 & 0,01 & 16,35 & 0,09 & 0,02 & 20,20 & 0,11 & 0,0001 & 0,0123 & 0,10 & 0,01 & 14,87 \\
\hline 1500 & 0,08 & 0,01 & 12,68 & 0,09 & 0,01 & 20,71 & 0,09 & 0,0008 & 0,0092 & 0,10 & 0,01 & 14,89 \\
\hline \multicolumn{13}{|c|}{$20-40 \mathrm{~cm}$ profundidade } \\
\hline 0,01 & 0,43 & 0,00 & 0,00 & 0,44 & 0,02 & 5,90 & 0,36 & 0,0007 & 0,027 & 0,43 & 0,00 & 0,00 \\
\hline 10 & 0,17 & 0,01 & 9,24 & 0,17 & 0,01 & 9,24 & 0,23 & 0,00211 & 0,0459 & 0,18 & 0,01 & 8,09 \\
\hline 100 & 0,14 & 0,01 & 8,29 & 0,12 & 0,01 & 10,79 & 0,17 & 0,00052 & 0,0229 & 0,12 & 0,01 & 8,24 \\
\hline 300 & 0,12 & 0,01 & 10,07 & 0,11 & 0,01 & 11,56 & 0,18 & 0,00474 & 0,068 & 0,11 & 0,01 & 8,69 \\
\hline 500 & 0,10 & 0,01 & 11,96 & 0,10 & 0,01 & 11,93 & 0,16 & 0,00371 & 0,0609 & 0,10 & 0,00 & 8,63 \\
\hline 1000 & 0,09 & 0,01 & 8,21 & 0,09 & 0,01 & 12,43 & 0,12 & 0,00019 & 0,0139 & 0,10 & 0,00 & 9,05 \\
\hline 1500 & 0,08 & 0,01 & 9,82 & 0,08 & 0,01 & 12,73 & 0,11 & 0,00033 & 0,0182 & 0,10 & 0,00 & 9,01 \\
\hline \multicolumn{13}{|c|}{$40-60 \mathrm{~cm}$ profundidade } \\
\hline 0,01 & 0,49 & 0,00 & 0,00 & 0,44 & 0,02 & 4,94 & 0,36 & 0,0009 & 0,030 & 0,49 & 0,00 & 0,00 \\
\hline 10 & 0,18 & 0,05 & 31,58 & 0,14 & 0,02 & 18,24 & 0,24 & 0,00137 & 0,0371 & 0,19 & 0,06 & 35,45 \\
\hline 30 & 0,15 & 0,05 & 31,57 & 0,12 & 0,02 & 20,61 & 0,21 & 0,00040 & 0,0202 & 0,14 & 0,044 & 30,01 \\
\hline 100 & 0,13 & 0,04 & 35,12 & 0,10 & 0,02 & 23,24 & 0,19 & 0,00036 & 0,0191 & 0,11 & 0,03 & 27,35 \\
\hline 300 & 0,10 & 0,03 & 29,75 & 0,08 & 0,02 & 25,67 & 0,17 & 0,00047 & 0,021 & 0,10 & 0,02 & 26,49 \\
\hline 500 & 0,09 & 0,01 & 21,32 & 0,07 & 0,02 & 26,80 & 0,13 & 0,00022 & 0,0149 & 0,09 & 0,02 & 27,17 \\
\hline 1000 & 0,08 & 0,01 & 23,40 & 0,07 & 0,02 & 28,35 & 0,11 & 0,00023 & 0,0154 & 0,09 & 0,02 & 26,47 \\
\hline 1500 & 0,07 & 0,01 & 24,99 & 0,06 & 0,01 & 29,26 & 0,10 & 0,00011 & 0,0107 & 0,08 & 0,02 & 26,17 \\
\hline
\end{tabular}


às variações nos valores da dimensão fractal e valores, tanto de $D_{\text {PSD }}$ e $D_{\text {SWRC }}$, menor que dois, poderão indicar problemas metodológicos na obtenção da curva de retenção.

Nota-se, pela Tabela 1, que as menores variações dos teores de água no solo ocorreram na presença de baixas tensões, entre os valores de 0,01 a $100 \mathrm{kPa}$ e maiores variações entre 300 a $1500 \mathrm{kPa}$, para os potenciais mais altos. Observa-se também, para as três profundidades do solo, que as maiores variações nos teores de água ocorreram na tensão que corresponde ao ponto de murcha permanente $(1500 \mathrm{kPa})$; referidos resultados corroboram com aqueles encontrados por Poulsen et al. (1999), que evidenciaram maior variabilidade compreendida entre as tensões de água no solo, de 200 a $1500 \mathrm{kPa}$.

A Figura 1 apresenta as curvas de retenção de água no solo ajustadas pelos três modelos avaliados (BC - Brooks \& Corey (1964); e $\mathrm{BCD}_{\mathrm{SWRC}}$ e $\mathrm{BCD}_{\mathrm{PSD}}$ - Brooks \& Corey (1964) modificados, respectivamente, para o tamanho dos poros do solo e para a distribuição do tamanho de suas partículas. Na mesma Figura se tem uma visualização melhor do comportamento dessa variabilidade dos valores médios dos teores de água no solo em função das tensões aplicadas ao solo.

Nota-se, pela Figura 1, que os modelos Brooks \& Corey

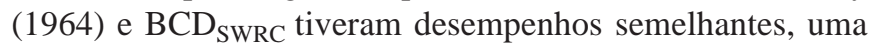
vez que estimaram valores de umidade bem próximos dos valores experimentais em virtude, provavelmente, do fato desses dois modelos expressarem o tamanho dos poros do

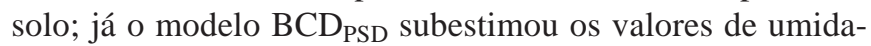
de quando o solo foi submetido a baixas tensões e os superestimou quando em altos potenciais matriciais, indicando que para o estudo de movimento de água no solo é mais adequado levar em consideração a geometria e o diâmetro médio dos poros do solo e não a distribuição dos tamanhos das partículas (Andrade, 2002).

Nos potenciais matriciais de 0 até $100 \mathrm{kPa}$, os modelos

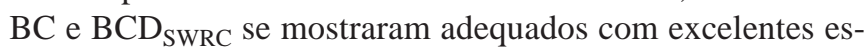
timativas, porém o modelo BC tende a subestimar os valores de $\theta$ com o acréscimo do potencial matricial; já o modelo $\mathrm{BCD}_{\text {SWRC }}$ subestima os valores de $\theta$ no intervalo de 100 a $300 \mathrm{kPa}$ estimando, entretanto, com boa precisão, o conteúdo de umidade do solo em potenciais superiores a $500 \mathrm{kPa}$ (500 a $1500 \mathrm{kPa}$ ) nas três profundidades estudadas; desta forma, a curva dos valores de $\theta$ estimada pelo modelo $\mathrm{BCD}_{\text {SwRC }}$ foi a que mais se aproximou da curva dos dados experimentais conferindo, então, melhor adequabilidade, em relação aos demais modelos testados (Figura 1), porém Tyler \& Wheatcraft (1990) encontraram boa concordância de resultados entre curvas de retenção estimadas pela dimensão fractal da distribuição de partículas $\left(\mathrm{D}_{\mathrm{PSD}}\right)$ e as determinadas em laboratório, principalmente para solos cujo valor de $\mathrm{D}$ é maior que 3 .

Na Tabela 2 se apresentam os valores dos parâmetros de erro padrão de estimativa da média (ES) utilizados para a avaliação da qualidade e para comparação do desempenho do modelo proposto por Books \& Corey (1964) modificado por Pierrier et al. (1996), na predição da curva de retenção de água no solo em relação aos valores medidos.

Tem-se, na Tabela 2, que o ajuste dos dados pelo modelo BCD $_{\text {PSD }}$ resultou em altos valores de 11,416, 23,275 e
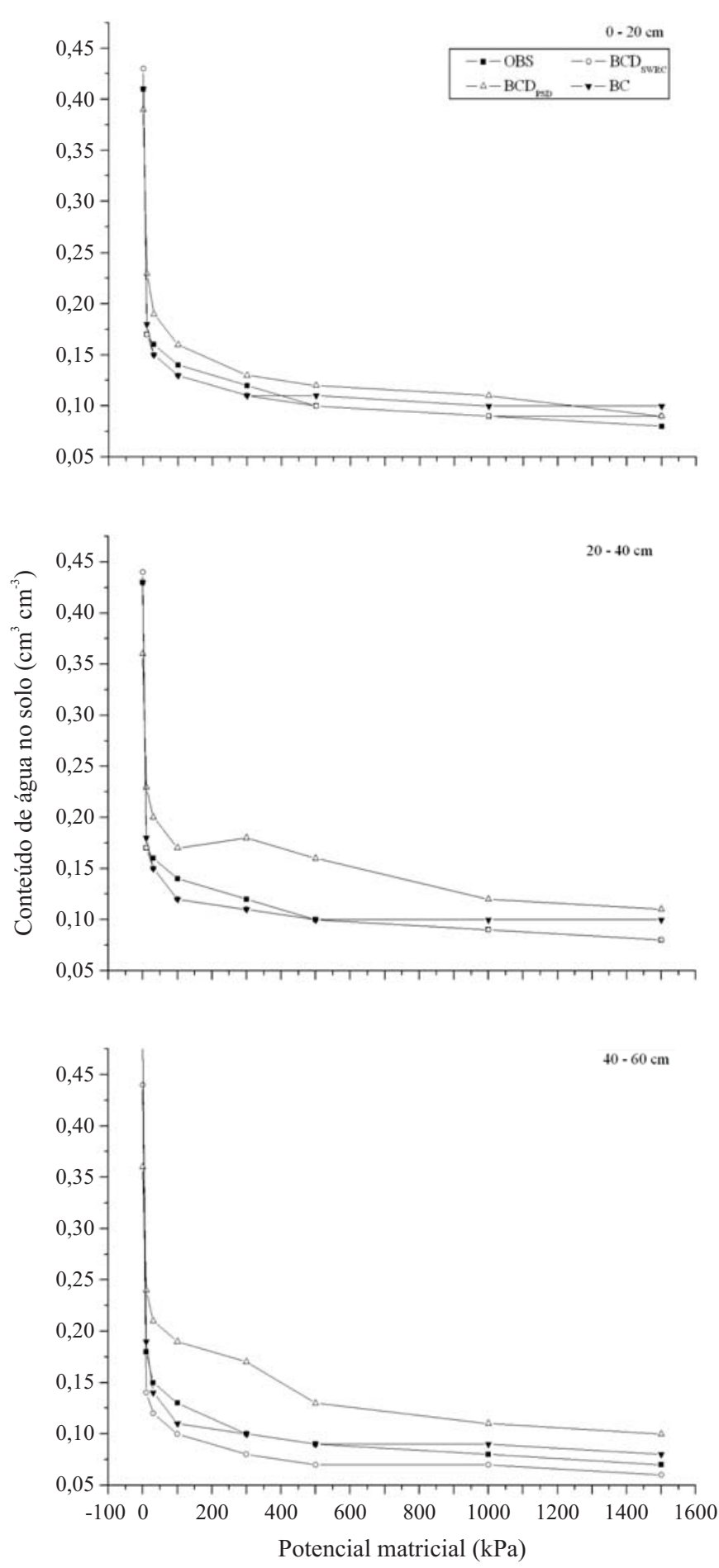

Figura 1. Curvas de retenção de água no solo ajustadas pelos três modelos avaliados em comparação com os valores observados nas profundidades de 0-20, 20-40 e 40-60 cm

Tabela 2. Erro padrão de estimativa para os modelos de ajuste da curva de retenção de água no solo nas três profundidades estudadas

\begin{tabular}{lccc}
\hline \multirow{2}{*}{ Modelos } & \multicolumn{3}{c}{ Erro padrão de estimativa } \\
\cline { 2 - 4 } BCD $_{\text {SWRC }}$ & $\mathbf{0 - 2 0} \mathbf{~ c m}$ & $\mathbf{2 0 - 4 0} \mathbf{~ c m}$ & $\mathbf{4 0 - 6 0 ~} \mathbf{~ m ~}$ \\
BCD $_{\text {PSD }}$ & 0,0136 & 0,0124 & 0,0435 \\
BC & 11,416 & 23,275 & 15,336 \\
\hline
\end{tabular}


15,336, para as profundidades de 0-20, 20-40 e 40-60 cm, respectivamente; já com os modelos $\mathrm{BC}_{\mathrm{SWRC}}$ e $\mathrm{BC}$, esses parâmetros foram inferiores em relação aos valores observados, cujos resultados confirmam que os modelos $\mathrm{BC}_{\text {SWRC }} \mathrm{e}$ BC ajustaram melhor os dados do conteúdo de água no solo, em função do potencial matricial $[\theta(\Psi)]$, mostrando-se satisfatórios quando comparados com os dados observados; tal comportamento está associado ao fato da variação de $\mathrm{D}_{\text {SWRC }}$ conter fatores físicos diretamente relacionados com a retenção de água no solo, como a distribuição dos tamanhos dos poros e o tamanho dos agregados do solo (Castrignanò \& Stelluti, 1999); este resultado evidencia que, além do potencial, $\mathrm{D}_{\text {SwRC }}$ é outra propriedade de interesse da física do solo que se apresenta como ferramenta eficientemente capaz de quantificar a retenção de água no solo.

Na Tabela 3 está descrito o resumo estatístico da água disponível para três profundidades estimadas pelos três modelos usados nesta pesquisa; verifica-se que os valores médios da água disponível para as três profundidades se apresentam normalmente distribuídos havendo um coeficiente de variação para os três modelos estudados dentro de uma faixa aceitável para solos.

Tabela 3. Resumo estatístico da água disponível determinada em laboratório e estimada pelo modelo original de Brooks \& Corey (1964) e modificado com as dimensões fractais $D_{P S D}$ e $D_{S W R C}$ para as três profundidades

\begin{tabular}{|c|c|c|c|}
\hline \multirow{2}{*}{ Parâmetros Estatísticos } & \multicolumn{3}{|c|}{ Profundidade (cm) } \\
\hline & $0-20$ & $20-40$ & $40-60$ \\
\hline \multicolumn{4}{|c|}{ Método de Laboratório } \\
\hline Média & 9,242 & 9,305 & 10,978 \\
\hline Desvio padrão (\%) & 1,598 & 1,126 & 5,543 \\
\hline Coeficiente de variação (\%) & 17,293 & 12,098 & 50,496 \\
\hline Erro padrão de estimativa (ES) & 0,266 & 0,187 & 0,923 \\
\hline \multicolumn{4}{|c|}{ Modelo $\mathrm{BCD}_{\text {SWRC }}$} \\
\hline Média & 8,371 & 8,622 & 7,949 \\
\hline Desvio padrão (\%) & 0,674 & 0,554 & 0,762 \\
\hline Coeficiente de variação (\%) & 8,048 & 6,421 & 9,587 \\
\hline Erro padrão de estimativa (ES) & 0,804 & 0,946 & 1,586 \\
\hline \multicolumn{4}{|c|}{ Modelo BCD ${ }_{P S D}$} \\
\hline Média & 3,754 & 2,117 & 1,951 \\
\hline Desvio padrão (\%) & 0,545 & 0,196 & 0,663 \\
\hline Coeficiente de variação (\%) & 14,508 & 9,240 & 33,978 \\
\hline Erro padrão de estimativa (ES) & 7,889 & 10,243 & 14,337 \\
\hline \multicolumn{4}{|c|}{ Modelo BC - Brooks \& Corey (1964) } \\
\hline Média & 8,525 & 8,538 & 10,325 \\
\hline Desvio padrão (\%) & 1,289 & 0,852 & 5,636 \\
\hline Coeficiente de variação (\%) & 3,600 & 2,400 & 16,500 \\
\hline Erro padrão de estimativa (ES) & 1,049 & 1,157 & 1,970 \\
\hline
\end{tabular}

Estatisticamente, os valores de água disponível apresentaram pequena variabilidade nas profundidades estudadas de 0-20 e 40-60 cm com um acréscimo mais acentuado para a profundidade de 40-60 cm (50,49\%), quando determinada pelo método de laboratório.

Para o modelo BCD $_{\text {SwRC }}$ e sem uso da dimensão fractal (BC) Brooks \& Corey (1964), verificaram baixos valores dos parâmetros estatísticos coeficiente de variação e desvio pa- drão, para as três profundidades estudadas porém, para o modelo de Brooks \& Corey (1964) com a dimensão fractal baseada na curva de distribuição de partículas (BCD $\left.\mathrm{PSD}_{\mathrm{PS}}\right)$, constata-se uma variabilidade maior, tornando-se mais acentuada na última profundidade, fato que pode estar associado à ampla variação da composição das percentagens dos separados texturais do solo, à variação da estrutura do solo e ao tamanho da amostra.

É possível observar melhores valores para o modelo de Brooks \& Corey (1964) com aproximação fractal baseada no tamanho dos poros do solo ( $\left.\mathrm{BCD}_{\mathrm{SWRC}}\right)$, mostrando-se satisfatórios quando comparados com os dados experimentais; desta forma, o modelo BCD $\mathrm{SWRC}_{\mathrm{SW}}$ se apresenta apropriado para estimativa da água disponível do solo, com erro padrão de estimativa de 0,804, 0,946 e 1,586\%, respectivamente, nas profundidades de 0-20, 20-40 e 40-60 cm (Tabela 3). Esta abordagem pode, ainda, ser utilizada como outra ferramenta eficiente para predizer a água disponível no solo, por meio de relações funcionais da dimensão fractal com o modelo de Brooks \& Corey (1964).

\section{CONCLUSÃO}

Dentre os modelos testados, o modelo de Brooks \& Corey (1964) modificado para a dimensão fractal determinada em laboratório a partir do tamanho dos poros do solo (BCD $\mathrm{SWRC}_{\mathrm{S}}$ ), foi o que melhor estimou o conteúdo de água no solo quando comparado com as medições de laboratório, seguido do modelo original de Brooks \& Corey (1964).

\section{LITERATURA CITADA}

Andrade, A. R. S. de. Aplicação da teoria fractal e da geoestatistica na estimativa da condutividade hidráulica saturada e do espaçamento entre drenos. Botucatu: UNESP/FCA, 2002.181p. Tese Doutorado

Antonino, A. C. D.; Ruiz, C. F.; Souza, E. S. de; Netto, A. M.; Jaramillo, R. A. Distribuição probabilística do fator de escala de dois solos do Estado da Paraíba. Revista Brasileira de Engenharia Agrícola e Ambiental, v.8, n.2/3, p.220-224, 2004.

Bacchi, O. O. S.; Reichardt, K.; Villa Nova, N. A. Fractal scaling of particle and pore size distributions and its relation to soil hydraulic conductivity. Science Agriculture Engineering, v.53, p.356-361, 1996.

Bird, N. R. A.; Bartoli, F.; Dexter, A. R. Water retention models for fractal soil structures. Journal of Soil Science, v.47, p.1-6, 1996.

Brooks, R. H.; Corey, A. T. Hydrdraulic properties of porus media Hidrol. Pap. 3. Fort Collins: Colorado State University, 1964. 180p.

Bussab, W. O.; Marettin, P. A. Estatística básica. 5.ed. São Paulo, Saraiva, 2004. 120p.

Carvalho, J. R. P. de; Dechen, S. C. F.; Dufranc, G. Variabilidade espacial da agregação do solo avaliada pela geometria fractal e geoestatística. Revista Brasileira de Ciência do Solo, v.28, n.1, p.1-9, 2004. 
Castrignanò, A.; Stelluti, M. Fractal geometry and geostatistics for describing the field variability of soil aggregation. Journal Agricultural Engineering Research, v.73, p.13-18, 1999.

Coelho Filho, M. A.; Coelho, R. D.; Gonçalves, A. C. A. Variabilidade de variáveis físico-hidrícas do solo em um pomar de lima ácida Tahiti, irrigada por microaspersão. Revista Brasileira de Engenharia Agrícola e Ambiental, v.6, p.239-246, 2001.

EMBRAPA - Empresa brasileira de Pesquisa Agropecuária. Centro Nacional de Pesquisa de Solos. Brasília: Sistema Brasileiro de Classificação de Solos, 1999. 412p.

Fuentes, C.; Antonino, A. C. D.; Netto, A. M.; Lira, C. B. O.; Cabral, J. S. P. Desenvolvimento de um modelo fractal para a estimativa da condutividade hidráulica de solos não saturados. Revista Brasileira de Ciência do Solo, v.29, n.1, p.1-17. 2005.

Fuentes, C.; Brambila, F.; Vauclin, M.; Parlange, J. Y.; Haverkamp, R. Modelación fractal de la conductividad hidráulica de los suelos no saturados. Ingeniería Hidráulica, v.16, p.119-137, 2001.

Gee, G. W.; Bauder, J. W. Particle size analysis. In: METHODS of Soil Analysis: part I, 2.ed. Madison: American Society of Agronomy, 1986. p.383-411. Monograph Agronomy 9

Guerra, H. C. Física dos solos, l.ed. Campina Grande: UFPB, 2002. 173p.
Hott, M. C.; Soares, V. P.; Ribeiro. C. A. Á. S.; Griffith. J. J. Análise fractal de textura usando um operador de Hurst em uma imagem TM/Landsat. Simpósio Brasileiro de Sensoriamento Remoto, 12, 2005, Goiânia. Anais... Goiânia: INPE, 2005. p.4089-4093.

Libardi, P. L. Dinâmica da água no solo. Piracicaba: ESALQ, 2000. 497p.

Mayr, T.; Jarvis, N. J. Pedotransfer function to estimate soil water retention parameters for a modified Brooks-Corey type model. Geoderma, v.91, p.1-9, 2000.

Perfect, E.; Rasiah, V.; Kay, B. D. Fractal dimension of soil aggregate-size distributions calculated by number and mass. Soil Science Society of America Journal, v.56, n.5, p.1407-1409, 1992.

Pierrier, E.; Rieu, M.; Sposito, G.; Marsily, G. Models of the water retention curve for soils with a fractal pore size distribution. Water Resources Research, v.32, p.3025-3031, 1996.

Poulsen, T. G.; Moldrup, P. Yamaguchi, T. Jacobsen, O. H. Predicting saturated and unsaturated hydraulic conductivity in undisturbed soils from soil water characteristics. Soil Science, v.164, n.12, p.877-887, 1999.

Tyler, S. W.; Wheatcraft, S. W. Fractal processes in soil water retention. Water Resources Research, v.26, p.1047-1054, 1990.

Tyler, S. W.; Wheatcraft, S. W. Fractal scaling of soil particle-size distributions: Analysis and limitations. Soil Science Society of America Journal, v.56, p.362-369, 1992. 